\title{
Is isomerism of the atrial appendages a real thing?
}

\author{
Robert H. Anderson
}

$\mathrm{T}$ HE ACCOMPANYING PAPER BY MORISHIMA AND HER colleagues from Tokyo Women's Medical College describes an important advance in our understanding of the etiology of congenital cardiac malformations. By immersing the abdomen and thorax of pregnant rats in water varying in temperature from 43.0 to $43.5^{\circ} \mathrm{C}$, they were able to induce in the offspring the constellation of lesions generally known as visceral heterotaxy. As the authors discuss, this has potential implications for the social habits of the Japanese, who often spend time in outdoor hot springs which have a temperature equal to or higher than $43^{\circ} \mathrm{C}$. As yet, however, there is no evidence to show that the incidence of visceral hererotaxy is higher in the offspring of those who indulge this habit or, indeed, that congenital heart malformations in general have a greater incidence in those with a penchant for hot baths!

The findings of the paper of Morishima et al, ${ }^{1}$ nonetheless, are of great interest from the stance of their anatomic observations. There is ongoing debate concerning the utility of the appendages as a marker of atrial arrangement ("situs"). My colleagues and I have long held that the syndrome of visceral heterotaxy, from the stance of the heart itself, is best analyzed initially according to the morphology of the appendages, thus permitting the description of the variants of right and left isomerism. ${ }^{2,3}$ This observation was by no means original, since Van Mierop and Wiglesworth, in a seminal paper, ${ }^{4}$ showed that not only the right atrial appendages, but also the sinus nodes are duplicated in most examples of asplenia. Van Praagh and his colleagues, in contrast, hold contrary views. ${ }^{5,6}$ In their opinion, the arrangement of the atrial appendages is insufficiently constant to serve as a marker of isomerism. They prefer to place all cases into the categories of solitus or inversus depending on the venoatrial connections. From my obviously biased stance, this seems to be a gross violation of the "morphologic method," which Van Praagh et $\mathrm{al}^{7}$ rightly established as the guide to the recognition of cardiac structures. This principle, in brief, stated that one variable feature should not be used as a criterion for

Accepted for publication 17 March 1995 definition of another feature. Yet this is precisely what happens when venoatrial connections, particularly variable in the setting of visceral heterotaxy, are used as the primary criterion for recognition of atrial situs.

Van Praagh et al, ${ }^{5,6}$ however, made other criticisms of the concept of isomerism. First, they pointed out that shape of the appendages varies markedly, and could be modified by size. Second, they suggested that a patient described as having left atrial isomerism might be construed as having eight pulmonary veins. Both of these criticisms are relevant to the study of Morishima et al.' The group from Tokyo Women's Medical College have used a different criterion for distinction of the morphologically right and left atrial chambers, namely the nature of the junction of the appendage with the rest of the atrium. They point out that, in a morphologically right atrium, the pectinate muscles of the appendage extend all round the vestibule of the atrioventricular valve. In contrast, in a morphologically left atrium the pectinate muscles are confined to the anterior quadrants of the vestibule. These are the criteria which we used ourselves in our analysis of mice with the iv mutation. ${ }^{8}$ We have now shown in an analysis of 183 specimens that the criteria are equally valuable in analysis of human hearts from patients with visceral heterotaxy (Uemura et al, unpublished observations). In terms of the description of isomerism of atrial appendages versus atrial chambers, it is a fact that the heart with left isom-erism never has eight pulmonary veins. It is more accurate, therefore, to speak of isomerism of the appendages. When I discussed this with Dr. Morishima while preparing this paper for publication, nonetheless, she responded to me: "...isomerism in our rat model included isomerism not only of the appendage but also of the atrial cavity." She pointed out to me, emphasizing a fact with which I am in total agreement, that the morphologically left atrium is readily recognized in the absence of pulmonary veins, as in the patient with usual atrial arrangement and totally anomalous pulmonary venous connection. And, while from my stance it seems more accurate to describe "isomerism of the atrial appendages," it is certain that "atrial isomerism" will continue to be used in a clinical context. Those who employ the term are well aware that the usage 
is based upon the fact that the junctions of the appendages with the atrial chambers, in these circumstances, are mirror-images of each other, making allowances for the fact that they are not always of the same size. We must now wait to see how useful are the morphologic criteria in the clinical setting. Using the feature of extent of the pectinate muscles certainly permits isomerism of the appendages to be recognized morphologically even in fetal rats ${ }^{1}$ and mice. ${ }^{8}$ It is my belief that the same feature, when sought for diligently, should be recognized with high quality cross-sectional echocardiography. Only time will tell whether this is borne out by clinical experience, and whether the extent of the pectinate muscles proves as useful a marker in humans as the Tokyo group have shown it to be in their exciting animal model.

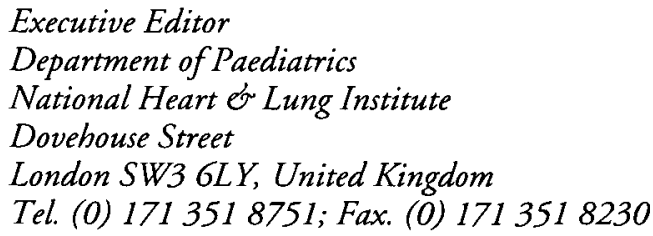

\section{References}

1. Morishima M, Miyagawa-Tomita S, Yasui H, Ando M,
Nakazawa M, Takao A. Visceroatrial heterotaxy syndrome induced by maternal hyperthermia in the rat. Cardiol Young 1995; 5: 251-256.

2. Macartney FJ, Zuberbuhler JR, Anderson RH. Morphological considerations pertaining to recognition of atrial isomerism: Consequences for sequential chamber localisation. $\mathrm{Br}$ Heart J 1980; 44: 657-667.

3. Sharma S, Devine W, Anderson RH, Zuberbuhler JR. The determination of atrial arrangement by examination of appendage morphology in 1842 heart specimens. Br Heart J $1988 ; 60: 227-231$.

4. Van Mierop LHS, Wiglesworth FW. Isomerism of the cardiac atria in the asplenia syndrome. Lab Invest 1962; 11 : 13031315.

5. Van Praagh S, Kakou-Guikahue M, Kim H-S, Becker J, Alday L, Van Praagh R. Atrial situs in patients with visceral heterotaxy and congenital heart disease: Conclusions based on findings in 104 postmortem cases. Coeur 1988; 19: 484-502.

6. Van Praagh R, Van Praagh S. Atrial isomerism in the heterotaxy syndromes with asplenia, or polysplenia, or normally formed spleen: an erroneous concept. Am J Cardiol 1990; 66: 15041506.

7. Van Praagh R, David I, Wright GB, Van Praagh S. Large RV plus small LV is not single RV. Circulation 1980; 61: 10571058.

8. Seo J-W, Brown NA, Ho SY, Anderson RH. Abnormal laterality and congenital cardiac anomalies: Relations of visceral and cardiac morphologies in the iv/iv mouse. Circulation 1992; 86: 642-650. 